\title{
ON A CLASS OF CONTINUOUS FLOW
}

\author{
RAMON MOGOLLON \\ Departamento de Matemáticas \\ Escuela de Ciencias \\ Universidad Centro Occidental \\ Barquisimeto, Venezuela
}

(Received August 20, 1979 and in revised form November 9, 1979)

ABSTRACT. This paper involves characterizations of a class of continuous flows. The flows considered are those in which the positive prolongation of each point coincides with the trajectory through the point. The characterizations are based on the theory of prolongation.

KEY WORDS AND PHRASES. F.LW, prolongation, trajectory, periodic, critical. 1980 MATHEMATICS SUBJECT CLASSIFICATION CODES. Primary 34 C35.

1. INTRODUCTION.

The purpose of this paper is to study dynamical systems in which the positive prolongation of each point coincides with the trajectory through the point. Ahmad [1] studied similar flows--flows in which the positive prolongation of each point coincides with the closure of the positive semi-trajectory through the point. He referred to such flows as flows of characteristic $0^{+}$. Other authors 
have studied these flows or similar ones (see [1], [2], [3], [5], [6]). Knight [6], for example, gave characterizations of flows of characteristic 0, i. e., flows in which the prolongation of each point coincides with the closure of the trajectory through the point. The author in [7] characterized flows in which the positive prolongation of each point coincides with the positive semi-trajectory through the point.

In this paper we give several characterizations of flows where the positive prolongation of each point coincides with the trajectory through the point. We show that several seemingly different flows are all equivalent, thus making it unnecessary to study all such flows. We also give a simple characterization of our flows for the case where the phase space is restricted to the two dimensional space $R^{2}$.

\section{DEFINITIONS AND NOTATIONS.}

We shall let $R, R^{+}, R^{-}$represent the real numbers, the non-negative real numbers, and the non-positive real numbers, respectively. By a dynamical system or a continuous flow is meant a pair $(X, \pi)$, where $X$ is a topological space, referred to as the phase space, and $\pi: X \times R \rightarrow \chi$ is a mapping satisfying the following three axioms:

$$
\begin{aligned}
& \pi(x, 0)=x, \\
& \pi(\pi(x, t), s)=\pi(x, t+s), \\
& \pi \text { is continuous. }
\end{aligned}
$$

For convenience, we shall let $\pi(x, t)$ be denoted by $x \cdot t$, or simply $x t$. For each $x \in X, C(x)=x \cdot R,\left(C^{+}(x)=x \cdot R^{+}\right),\left(C^{-}(x)=x \cdot R^{-}\right.$) represents the trajectory (positive semitrajectory) (negative semitrajectory) through $x$. A point $x$ in $x$ is called a critical point if $x t=x$ for all $t$ in $R$. A non-critical point is called a periodic point if there exists a number $t, t>0$, in $R$ satisfying $x t=x . A$ 
subset $M$ of $X$ is said to be positively invariant (negatively invariant) (invariant) if $C^{+}(M)=M\left(C^{-}(M)=M\right)(C(M)=M)$. For each $x$ in $X$, we let $K(x)=$ $\overline{C(x)}, K^{+}(x)=\overline{C^{+}(x)}$, and $K^{-}(x)=\overline{C^{-}(x)}$. The positive 1imit set of a point $x$ in $X$ is denoted by $L^{+}(x)$, i. e., $L^{+}(x)=\left\{y \in X: x t_{i} \rightarrow y\right.$ for some net $\left(t_{i}\right)$ of $\mathrm{R}^{+}$with $t_{i} \rightarrow+\infty$. Similarly, the negative limit set and the limit set of $x$ are denoted by $\mathrm{L}^{-}(\mathrm{x})$ and $\mathrm{L}(\mathrm{x})$, respectively. The positive prolongation of a point $x$ of $x$ is denoted by $D^{+}(x)$, i.e., $D^{+}(x)=$ fy $\varepsilon x$ : there exist nets $\left(x_{i}\right)$ of $x$ and $\left(t_{i}\right)$ of $R^{+}$such that $\left.x_{i} \rightarrow x, x_{i} t_{i} \rightarrow y\right\}$. Similarly, $D^{-}(x)$ and $D(x)$ denote the negative prolongation and the prolongation of $x$, respectively. The positive prolongational limit set of a point $x$ in $x$ is denoted by $\mathrm{J}^{+}(x)$, i.e.,

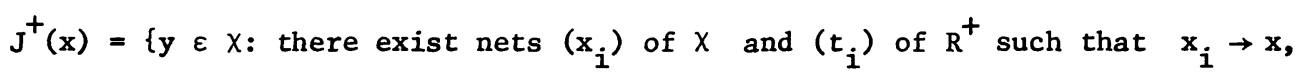
$\left.t_{i} \rightarrow+\infty, x_{i} t_{i} \rightarrow y\right\}$. Similarly, $J^{-}(x)$ and $J(x)$ denote the negative prolonga$\underline{\text { tional }} \underline{\text { limit set }}$ and the prolongational limit set, respectively. A point $x$ in $X$ is said to be positively poisson stable if $x \in L^{+}(x)$. The negative and bilateral versions are defined similarly. A point $\mathbf{x}$ in $X$ is said to be nonwandering if $x \in J^{+}(x)$. A point $x$ in $X$ is said to be positively dispersive if $\mathrm{J}^{+}(\mathrm{x})=\emptyset$. The negative and bilateral versions are defined similarly. For more information on the above concepts and related notions pertinent to this paper one is referred to the bibliography.

Throughout this paper, the phase space is always assumed to be Hausdorff.

3. CHARACTERIZATIONS OF FLOWS SATISFYING $\mathrm{D}^{+}(\mathrm{x})=\mathrm{C}(\mathrm{x})$.

THEOREM 3.1. Let $(X, \pi)$ be any continuous flow. Then the following conditions are equivalent:

(1) $D^{+}(x)=C(x)$ for all $x$ in $x$.

(2) $\mathrm{J}^{+}(\mathrm{x})=\mathrm{C}(\mathrm{x})$ for all $\mathrm{x}$ in $x$.

(3) $J^{-}(x)^{\prime}=c(x)$ for all $x$ in $x$. 
(4) $J(x)=C(x)$ for all $x$ in $x$.

(5) $D(x)=C(x)$ for all $x$ in $X$, and there are no positively dispersive points.

(6) $D^{-}(x)=C(x)$ for all $x$ in $x$.

PROOF. Assume that (1) holds. Let $x \in X$. Then $\mathrm{J}^{+}(\mathrm{x}) \neq \emptyset$. For, $\mathrm{J}^{+}(\mathrm{x})=\emptyset$ implies that $\mathrm{D}^{+}(\mathrm{x})=\mathrm{C}^{+}(\mathrm{x}) \cup \mathrm{J}^{+}(\mathrm{x})=\mathrm{C}^{+}(\mathrm{x})$. It follows from (1) that $\mathrm{c}^{+}(x)=$ $c(x)$. Therefore, $x \cdot(-1)=x \cdot t$ for some $t \in R^{+}$, and hence $x \cdot(t+1)=x$. This shows that $x$ is either a critical or a periodic point; in either case $\mathrm{L}^{+}(x)=$ $\mathrm{C}^{+}(\mathrm{x}) \neq \emptyset$ implies that $\mathrm{J}^{+}(\mathrm{x}) \neq \emptyset$, which contradicts the assumption that $\mathrm{J}^{+}(\mathrm{x})=$ ø. We note that $\mathrm{J}^{+}(x) \subset \mathrm{D}^{+}(x)=\mathrm{C}(\mathrm{x})$. But, since $\mathrm{J}^{+}(x)$ is an invariant set, we must also have $c(x) \subset J^{+}(x)$. Hence, $J^{+}(x)=c(x)$, and (2) holds.

Assume that (2) holds. Let $x \in X$. Then $x \in J^{+}(x)$. Hence, $x \in J^{-}(x)$ since, in general, $x \in J^{+}(y)$ implies $y \in J^{-}(x)$ for any two points $x$, $y$ in $x$. Since $\mathrm{J}^{-}(\mathrm{x})$ is invariant, we have $\mathrm{C}(\mathrm{x}) \subset \mathrm{J}^{-}(\mathrm{x})$. Now, let $\mathrm{y} \in \mathrm{J}^{-}(\mathrm{x})$. Then $x \in \mathrm{J}^{+}(\mathrm{y})=$ $c(y)$, and hence $y \in c(x)$. This shows that $J^{-}(x) \subset c(x)$. Therefore, $J^{-}(x)=$ $C(x)$, and (3) holds.

Assume that (3) holds. Let $x \in X$. Then $C(x)=J^{-}(x) \subset J(x)$. Now, let $y \in \mathrm{J}^{+}(\mathrm{x})$. Then, $\mathrm{x} \in \mathrm{J}^{-}(\mathrm{y})=\mathrm{C}(\mathrm{y})$, and hence $\mathrm{y} \in \mathrm{C}(\mathrm{x})$. This shows that $\mathrm{J}^{+}(\mathrm{x})$ $\subset C(x)$. Hence, we have $J(x)=J^{+}(x) \cup J^{-}(x) \subset C(x)$. Consequently, $J(x)=$ $C(x)$, and (4) holds.

Assume that (4) holds. Let $x \in X$. Obviously, $D(x)=C(x)$, since $D(x)=$ $C(x) \cup J(x)$. To see that $x$ is not positively dispersive, i. e., $J^{+}(x) \neq \emptyset$, we note that by $(4), J^{+}(x)=\emptyset$ implies that $x \in J^{-}(x)$. But $x \in J^{-}(x)$ implies that $\mathrm{x} \in \mathrm{J}^{+}(\mathrm{x})$, contradicting $\mathrm{J}^{+}(\mathrm{x})=\emptyset$. Thus, (5) holds.

Assume that (5) holds. Let $x \in x$. Then, $\emptyset \neq \mathrm{J}^{+}(\mathrm{x}) \subset \mathrm{D}(\mathrm{x})=\mathrm{C}(\mathrm{x})$. Since $\mathrm{J}^{+}(x)$ is a nonempty invariant subset of $\mathrm{C}(\mathrm{x})$, we must have $\mathrm{C}(x) \subset \mathrm{J}^{+}(x)$. Hence, 
$x \in J^{+}(x)$, and, consequently, $x \in J^{-}(x)$. This shows that $c(x) \subset J^{-}(x)$. Therefore, $C(x) \subset D^{-}(x)$ since $J^{-}(x) \subset D^{-}(x)$. It is obvious from (5) that $D^{-}(x) \subset$ $C(x)$. Thus, we have $D^{-}(x)=C(x)$, and (6) holds.

Finally, assume that (6) holds. Let $x \in X$. We note that $J^{-}(x)=\emptyset$ would imply that $C^{-}(x)=C(x)$ since $D^{-}(x)=C^{-}(x) \cup J^{-}(x)$. But, as was shown earlier, $C^{-}(x)=c(x)$ would imply that $x$ is a critical or a periodic point, thus contradicting $\mathrm{J}^{-}(x)=\emptyset$. Thus, we have $\emptyset \neq \mathrm{J}^{-}(x) \subset \mathrm{D}^{-}(x)=\mathrm{C}(\mathrm{x})$. Again, since $\mathrm{J}^{-}(\mathrm{x})$ is invariant, we must have $c(x) \subset J^{-}(x)$. This implies that $x \in J^{-}(x)$, and hence, $x \in J^{+}(x)$. Therefore, $c(x) \subset J^{+}(x) \subset D^{+}(x)$. Now, let $y \in D^{+}(x)$. Then, $x \in D^{-}(y)=C(y)$. But, $x \in C(y)$ implies that $y \in C(x)$. This shows that $D^{+}(x)$ $C C(x)$. Therefore, $D^{+}(x)=C(x)$, and hence (1) holds. This completes the proof of the theorem.

We note that if $D(x)=C(x)$, then $D(x)=K(x)$ since $C(x) \subset K(x) \subset D(x)$. Thus, the equivalence of statements (1) and (5) in the preceding theorem shows that the class of flows that we are studying here form a subclass of flows of characteristic 0 (recall [6] that a flow is said to have characteristic 0 if $D(x)=K(x)$ for all $x$ in the phase space). In addition, we showed in the preceding theorem that $\mathrm{D}^{+}(x)=\mathrm{C}(\mathrm{x})$ implies that $\mathrm{J}^{+}(x) \neq \emptyset$. Since $\mathrm{J}^{+}(x)$ is invariant, we must have $c(x) \subset J^{+}(x)$. Therefore, we have

COROLLARY 3.2. If $\mathrm{D}^{+}(\mathrm{x})=\mathrm{C}(\mathrm{x})$ for each $\mathrm{x}$ in $\mathrm{X}$, then the flow is a nonwandering flow of characteristic 0 .

EXAMPLE 3.3. Consider the flow $\left(R^{2}, \pi\right)$ defined by the system of differential equations

$$
\begin{aligned}
& \dot{\mathbf{x}}=1 \\
& \dot{\mathbf{y}}=0
\end{aligned}
$$

It is easy to verify that this flow satisfies the condition $D(x)=C(x)$ for 
all $x$ in $R^{2}$ and is of characteristic 0 . But any point $x$ has the property $D^{+}(x)$ $\neq C(x)$. This shows that our flows form a proper subclass of flows of characteristic 0 .

REMARK 3.4. A trivial example of a flow of characteristic 0 that satisfies the condition $D^{+}(x)=C(x)$ is a global Poincaré center.

THEOREM 3.5. Let $(X, \pi)$ be a dynamical system where the phase space $X$ is either a locally compact metric space or a complete metric space. Further, assume that $D^{+}(x)=C(x)$ for each $x$ in $X$. Then the set of periodic and critical points is dense in $X$.

PROOF. Obviously, if $x$ is either periodic or critical, then $x \in C(x)=$ $L^{+}(x)=L^{-}(x)$. On the other hand, suppose that there is a point $x$, which is Poisson stable but neither periodic nor critical. If $x \in \mathrm{L}^{+}(x)$, then since $\mathrm{L}^{+}(\mathrm{x})$ is invariant, we have $\mathrm{C}(\mathrm{x}) \subset \mathrm{L}^{+}(\mathrm{x}) \subset \mathrm{D}^{+}(\mathrm{x})=\mathrm{C}(\mathrm{x})$. Therefore, $\mathrm{L}^{+}(\mathrm{x})=$ $C(x)$. But this contradicts a known result (see, e. g., [4]) that if $X$ is either locally compact and metric or complete metric, then every point that is neither periodic nor critical satisfies $\overline{L^{+}(x)-C(x)}=L^{+}(x)$. A similar argument shows that if $\mathrm{x} \in \mathrm{L}^{-}(\mathrm{x})$, then $\mathrm{x}$ is either periodic or critical. Thus, we have shown that the set of points that are either periodic or critical coincides with the set of Poisson stable points. We further note that by Corollary 3.2, all points of $X$ are non-wandering. The proof of our theorem now follows from a known result (see, e. g., [4]) that if the phase space $X$ is either locally compact and metric or complete metric, and if all points are nonwandering, then the set of Poisson stable points is dense in $X$. 
THEOREM 3.6. Let $(X, \pi)$ be any dynamical system, where, $X \subset R^{2}$. Then $\mathrm{D}^{+}(\mathrm{x})=\mathrm{C}(\mathrm{x})$ for all $\mathrm{x}$ in $\mathrm{X}$ if and only if the flow has characteristic 0 and there are no positively dispersive points.

PROOF. The "only if" part follows from Corollary 3.2. Now, suppose that the flow has characteristic 0 and $\mathrm{J}^{+}(\mathrm{x}) \neq \emptyset$ for each $\mathrm{x}$ in $\mathrm{X}$. We have $\mathrm{J}^{+}(\mathrm{x})$ $C D(x)=K(x)$. First let us assume that $L(x)=\emptyset$. Then, $K(x)=C(x)$, and hence $\mathrm{J}^{+}(\mathrm{x}) \subset \mathrm{C}(\mathrm{x})$. But $\emptyset \neq \mathrm{J}^{+}(\mathrm{x}) \subset \mathrm{C}(\mathrm{x})$ implies that $\mathrm{C}(\mathrm{x}) \subset \mathrm{J}^{+}(\mathrm{x})$. Hence, $\mathrm{J}^{+}(\mathrm{x})=$ $\mathrm{C}(\mathrm{x})$, which implies that $\mathrm{D}^{+}(\mathrm{x})=\mathrm{C}(\mathrm{x})$. Now, let us assume that $\mathrm{L}(\mathrm{x}) \neq \emptyset$. Let $y \varepsilon \mathrm{L}(\mathrm{x})$. Then $\mathrm{y} \varepsilon \mathrm{J}(\mathrm{x})$ and, hence, $\mathrm{x} \varepsilon \mathrm{J}(\mathrm{y}) \subset \mathrm{D}(\mathrm{y})=\mathrm{K}(\mathrm{y})$. But, since $\mathrm{L}(\mathrm{x})$ is a closed invariant set, we must have $K(y) \subset L(x)$. Therefore, $x \in L(x)$. Hence $\mathbf{x}$ is either a critical or a periodic point. This implies that $\mathrm{D}(\mathrm{x})=\mathrm{K}(\mathrm{x})=$ $\mathrm{C}(\mathrm{x})$. Thus, $\mathrm{D}^{+}(\mathrm{x}) \subset \mathrm{C}(\mathrm{x})=\mathrm{L}^{+}(\mathrm{x}) \subset \mathrm{D}^{+}(\mathrm{x})$. This shows that $\mathrm{D}^{+}(\mathrm{x})=\mathrm{C}(\mathrm{x})$, and the proof is complete.

NOTATION. Let $\left(R^{2}, \pi\right)$ be any continuous flow and $S$ be the set of critical points. For any $s \in S$ we shall hence forth let

$$
N_{s}=\left\{x \in R^{2}: x=s \text { or } x \text { is periodic and } S \cap \text { int } C(x)=\{s\}\right\}
$$

REMARK 3.7. Knight showed in [5] that there are six basic types of planar flows having characteristic 0 . These are

(1) parallelizable flows,

(2) flows having a global Poincaré center,

(3) flows where $S$ consists of one local Poincaré center $s, N_{s}$ is unbounded and $\partial \mathrm{N}_{s}$ is a single trajectory. The restriction of the flow to $\mathrm{R}^{2}-\mathrm{N}_{\mathrm{s}}$ is parallelizable,

(4) flows similar to Example 3 of [5],

(5) flows similar to Example 3 of [5], except that $\partial \mathrm{N}_{\mathbf{s}_{1}}=\partial \mathrm{N}_{\mathrm{s}_{2}}$ where 


$$
S=\left\{s_{1}, s_{2}\right\} \text {, and }
$$

(6) flows having only critical points.

From this and from theorem 3.6, it follows that there are three basic types of flows $\left(R^{2}, \pi\right)$ satisfying $D^{+}(x)=C(x)$ for each $x \in R^{2}$. These are the flows in (2), (5) and (6).

The author is grateful to Professor Shair Ahmad for suggesting this problem. His guidance and suggestions have been invaluable. The author also thanks the referee for his appropiate suggestions concerning the revision of this paper.

\section{References}

1. Ahmad, Shair, "Dynamical Systems of Characteristic $0^{+", ~ P a c . ~} \underline{\text { Jour. }}$ of Math. 32 $(1970), 561-574$.

2. Ahmad, Shair, "Strong Attraction and Classification of Certain Continuous Flows", Math Systems Theory $\underline{5}$ (1971), 157 - 163.

3. Bhatia, Nam, "Criteria for Dispersive Flows", Math. Nachr. $\underline{32}$ (1960), 89 - 93.

4. Bhatia, Nam and Hajek, Otmar, Theory of Dynamical Systems, Parts I and II, Technical Notes BN-599 and BN-606, University of Maryland, 1969.

5. Knight, Ronald, "Dynamical Systems of Characteristic 0", Pacific J. Math. 41 (1972), 447 - 457.

6. Knight, Ronald, "Structure and Characterizations of Certain Continuous Flows", Funkcial. Ekvac. 17 (1974), 223 - 230.

7. Mogollón, Ramón, "Characterizing Certain Continuous Flows", to appear in J. Math. and Phys. Sc.

8. Seibert, Peter and Tulley, Patricia, "On Dynamical Systems in the Plane", Arch. Math. 18 (1967), 290 - 292.

9. Ura, Taro, "Sur le Courant Extérieur a une Région Invariante; Prolongements d'une Caracteristique et l'order de Stabilité", Funkcial. Ekvac. 2 (1959), $143-200$. 


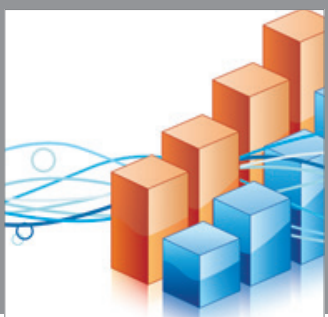

Advances in

Operations Research

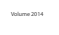

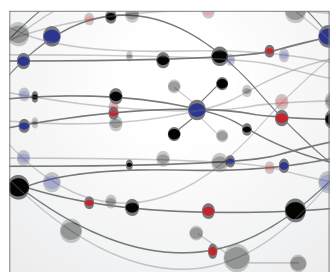

\section{The Scientific} World Journal
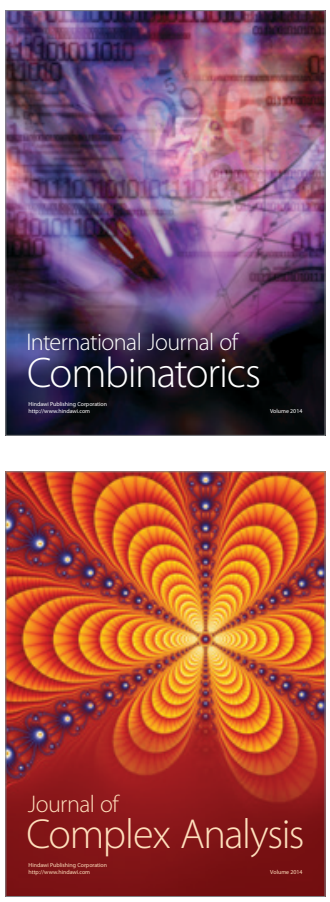

International Journal of

Mathematics and

Mathematical

Sciences
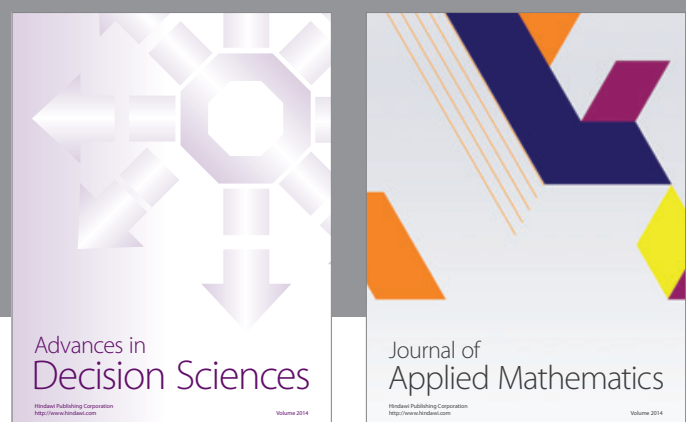

Journal of

Applied Mathematics
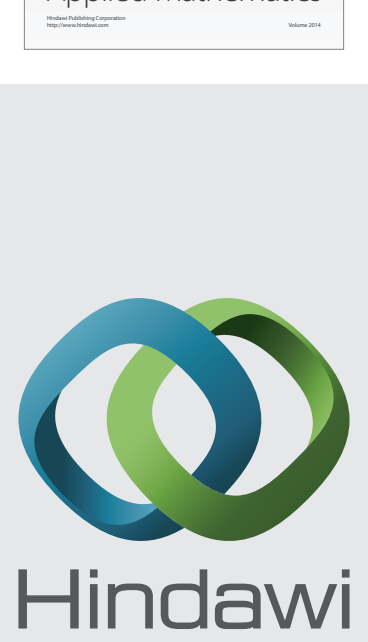

Submit your manuscripts at http://www.hindawi.com
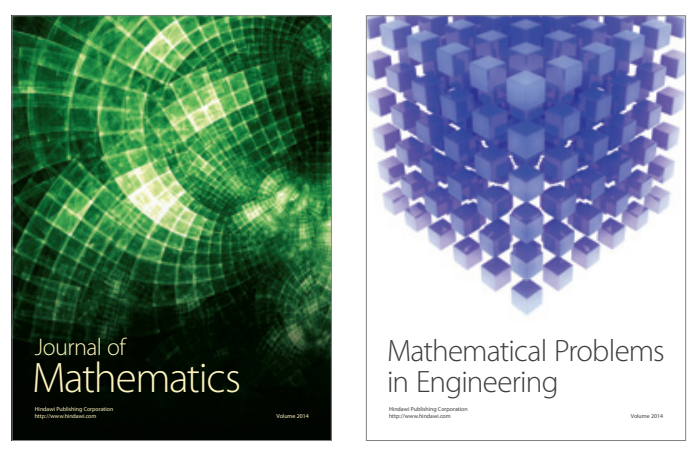

Mathematical Problems in Engineering
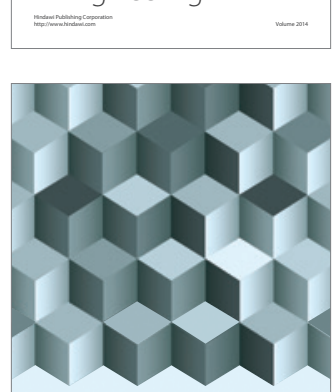

Journal of

Function Spaces
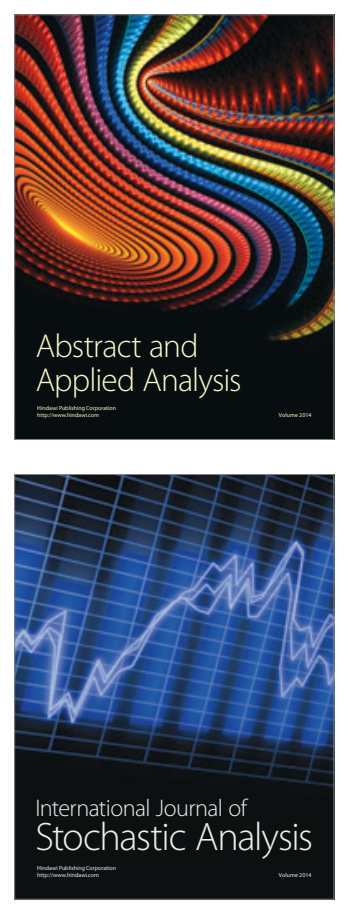

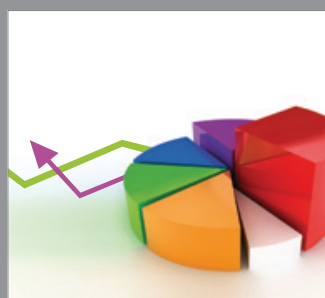

ournal of

Probability and Statistics

Promensencen
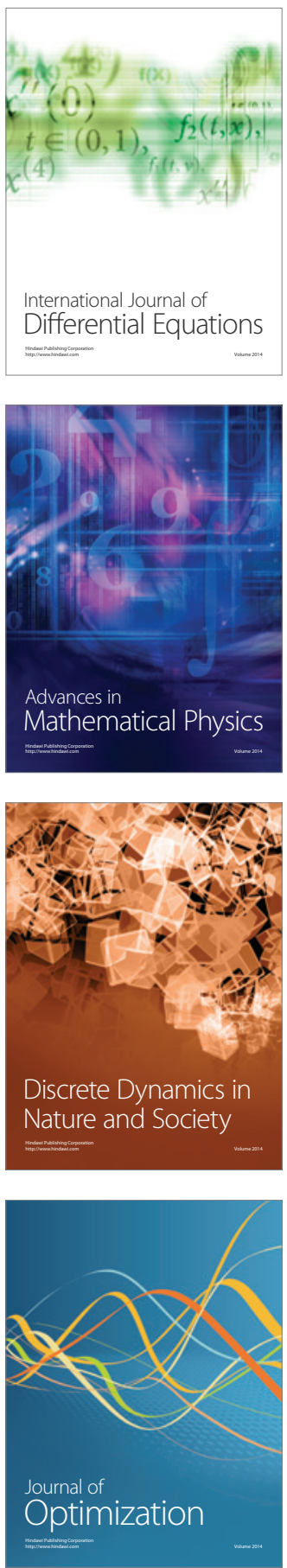